\title{
Situación actual de los Centros Municipales de Recuperación de Residuos Valorizables (CRRV) en Costa Rica
}

\author{
Current situation of Municipal Material \\ Recovery Facilities (MRF) in Costa Rica
}

Keneth Masís-Leandro' ${ }^{1}$, Lilliana Abarca-Guerrero²

Masís-Leandro, K; Abarca-Guerrero, L. Situación actual de los Centros Municipales de Recuperación de Residuos Valorizables (CRRV) en Costa Rica. Tecnología en Marcha. Vol. 32-4. Octubre-Diciembre 2019. Pág 3-17.

doi) https://doi.org/10.18845/tm.v32i4.4785

1 Escuela de Química, Instituto Tecnológico de Costa Rica. Costa Rica. Ingeniero Ambiental, Investigador principal con licenciatura en Ingeniería Ambiental. Correo electrónico: masisleandrokenneth@gmail.com. (iD) https://orcid.org/0000-0003-0946-973X

2 Escuela de Química, Instituto Tecnológico de Costa Rica. Costa Rica. Química, docente e investigadora. Departmento de Built Environment, Eindhoven University of Technology, Den Dolech, 25612 AZ Eindhoven, Holanda. Correo electrónico: labarca@tec.ac.cr. 


\title{
Palabras clave
}

Gestión Integral de Residuos Sólidos; Costa Rica; Municipalidad; Centro de Recuperación de Residuos Valorizables; Infraestructura; Tecnología; Procedimientos de trabajo.

\section{Resumen}

La promoción de Centros de Recuperación de Residuos Valorizables (CRRV) forma parte de la estrategia país para aumentar el porcentaje de residuos valorizados. Sin embargo, existe un vacío de conocimiento sobre el manejo y los procesos productivos de los CRRV a nivel nacional. Este trabajo explora los factores técnicos y ambientales relacionados con la infraestructura, tecnología y procedimientos de trabajo de los CRRV de propiedad municipal. Se obtuvo información a través de una encuesta de 15 CRRV municipales, de los cuales 5 fueron visitados para corroborar y ampliar la información recolectada. En el $80 \%$ de las instalaciones se procesan menos de 41 ton / mes de residuos y en el 93\% la operación de separación se lleva a cabo manualmente sobre una superficie estática, este método de separación es adecuado para la cantidad de residuos procesados en $87 \%$ de los casos. La ubicación, topografía y accesibilidad de los terrenos son en general adecuadas y no afectan negativamente el desempeño de las instalaciones. En la mayoría de los casos la infraestructura no fue diseñada para funcionar como CRRV, por lo que sus características no están orientadas a una máxima eficiencia en la operación. La ausencia de registros y procedimientos de trabajo escritos es una constante en los casos estudiados. En la mayoría de las instalaciones, se identificaron condiciones ergonómicas inadecuadas en las estaciones de clasificación, ausencia de métodos para evaluar el desempeño de los operarios y deficiencias en el proceso de capacitación del personal.

\section{Keywords}

Integrated Solid Waste Management; Costa Rica; Municipality; Material Recovery Facility; Infrastructure; Technology; Work procedures.

\begin{abstract}
The promotion of Material Recovery Facilities (MRF) is part of the country strategy to increase the percentage of recovered waste. However, there is a knowledge gap on the management and production processes of the MRF at the national level. This work explores the technical and environmental factors related to the infrastructure, equipment and work procedures of municipally owned MRF in the country. Information of 15 municipal MRF was obtained via a survey, of which 5 were visited in order to corroborate and expand the information collected. In $80 \%$ of the facilities, less than 41 ton / month of waste are processed and in 93\% of them the separation operation is carried out manually on a static surface, this separation method is suitable for the quantity of waste processed in $87 \%$ of cases. The location, topography and accessibility of the lands are generally adequate and do not negatively affect the performance of the facilities. In most cases, the infrastructure was not designed to function as a MRF, so its characteristics are not oriented to maximum efficiency in the operation. The absence of records and written work procedures is a constant in the facilities studied. In most cases, inadequate ergonomic conditions were identified in the classification stations, absence of methods to evaluate the performance of the operators and deficiencies in the personnel training process.
\end{abstract}




\section{Introducción}

La gestión de residuos sólidos con énfasis en la recuperación y valorización se ha convertido en una tendencia global [1]. Como parte de ese proceso, los Centros de Recuperación de Residuos Valorizables (CRRV) han pasado a jugar un rol central en los sistemas actuales de gestión de residuos. Los CRRV fungen como un paso intermedio entre la recolección de residuos a los generadores y su valorización en la industria, lo que ha permitido aumentar la cantidad de materiales recuperados y la reducción de los costos de toda la cadena de manejo [2], [3].

En Costa Rica, diversas leyes y reglamentos promueven y regulan el establecimiento de los CRRV. La Ley N 8839 para la Gestión Integral de los Residuos Sólidos [4] dispuso, en su artículo 8, el deber de las municipalidades de garantizar que en su territorio se brinde el servicio de centros de recuperación de materiales. Además, en la Estrategia Nacional de Separación, Recuperación y Valorización de Residuos (ENSRVR) [5] se establece que las autoridades locales deben valorar la construcción de esta clase de instalaciones. En la actualidad, según datos de la Fundación para la Sostenibilidad y la Equidad (Aliarse), un 30,4\% de las municipalidades del país cuenta con CRRV propio [6].

Los CRRV son instalaciones industriales y por lo tanto su eficiente funcionamiento depende de que se controlen cuidadosamente todos los aspectos de su diseño y operación [7]. Muchas municipalidades en Costa Rica enfrentan dificultades en los diversos aspectos del manejo de sus CRRV. En el aspecto financiero es común una asignación presupuestaria insuficiente para la Gestión Integral de Residuos Sólidos (GIRS), en lo institucional se tiene el riesgo de que las autoridades municipales de cada periodo tengan visiones distintas sobre la GIRS en el cantón y se dificulte la consolidación de proyectos. Adicionalmente, la urgente necesidad ambiental y de salud pública de recolectar y tratar una creciente generación de residuos puede provocar que las autoridades se decidan por la disposición final antes que la valorización. En el aspecto técnico es frecuente la ausencia de infraestructura adecuada para albergar un eficiente CRRV e insuficientes vehículos disponibles para nutrirlo con la recolección selectiva. En lo relativo al aspecto sociocultural, la cultura y el conocimiento de separar adecuadamente los residuos aún no son generalizados en la población. Finalmente, en el aspecto legal, no existe claridad en la implementación de sanciones e incentivos para promover la reducción y la valorización de residuos desde el origen [8], [9], [10].

En este contexto, el objetivo de este artículo es brindar información sobre la situación actual de algunos CRRV de propiedad municipal en Costa Rica, enfocándose en los aspectos técnicos y ambientales.

La revisión de literatura nacional e internacional permitió identificar los factores que desde el diseño y la operación afectan el desempeño técnico y ambiental de los CRRV. Los factores fueron agrupados basándose en lo hecho para la Illinois Recycling Association [11] y en su relación con las áreas funcionales de una empresa [12]. Alrededor de esta categorización se realizó la investigación y se presentan los resultados. Los grupos son los siguientes:

1. Localización y características del terreno donde se ubica el CRRV

2. Infraestructura disponible

3. Equipo y mobiliario disponible

4. Gestión de la materia prima

5. Gestión del recurso humano

6. Procedimientos de trabajo 


\section{Metodología}

Se elaboró una encuesta con el propósito de caracterizar a los CRRV respecto a los factores identificados en la literatura nacional e internacional. Se combinaron preguntas de respuesta corta, selección binomial (Sí/No), escala Likert de 5 puntos y casillas de verificación, según la información que se pretendiera recolectar.

Antes de ser enviada por correo electrónico, la encuesta se aplicó presencialmente en el CRRV de Montes de Oca, con el fin de evaluar su claridad, medir el tiempo de respuesta e introducir las modificaciones pertinentes. El instrumento fue enviado vía correo electrónico a 76 de las 81 municipalidades del país.

Se recibieron 15 encuestas completas. Posteriormente con la recomendación del señor MSc. Luis Diego Jiménez, Director de Proyectos Ambientales de la Fundación Aliarse [6] se seleccionaron cinco CRRV para realizar visitas de campo con el fin de corroborar y ampliar la información recibida en la encuesta.

Una vez procesadas las encuestas y realizadas las visitas a los CRRV se convocó a los encargados de los CRRV municipales a un taller participativo en las instalaciones del Instituto Tecnológico de Costa Rica. El objetivo del taller fue presentar los resultados parciales de la investigación y obtener el punto de vista de los presentes sobre la situación descrita. La metodología para preparar y conducir el taller se basó en el artículo ¿Cómo hacer talleres participativos con respuestas individuales? [13].

\section{Resultados y discusión}

Mediante la encuesta enviada por correo electrónico se obtuvo información de quince CRRV pertenecientes a municipalidades ubicadas en las regiones socioeconómicas Chorotega, Brunca y Central. La mayoría de las instalaciones de la región Central se ubican en el Gran Área Metropolitana (GAM). Por lo anterior, el estudio presenta una limitación ya que los resultados se refieren principalmente a CRRV de instalaciones ubicadas en la región central y metropolitana del país.

No se obtuvo autorización por parte de los municipios para nombrar explícitamente el cantón de los CRRV participantes del estudio, por lo cual se hará referencia a las instalaciones con un número, tal como aparece en el cuadro 1 junto con la región socioeconómica en que están ubicados y los años que llevan en operación.

Como puede observarse en el cuadro 1, doce de los CRRV estudiados se ubican en la región central del país, incluyendo diez de los treinta y un cantones que conforman la GAM, por lo tanto, los resultados de la presente investigación deben entenderse principalmente como reflejo de la situación de esta región del país. Resalta el hecho de que siete de los CRRV iniciaron operaciones antes de la promulgación de la Ley No 8839 [4]. En el caso de tres de las instalaciones visitadas esto se debe a que antiguamente eran instalaciones privadas que fueron adquiridas por el municipio.

\section{Localización y características del terreno donde se ubica el CRRV}

En general las instalaciones estudiadas se encuentran en terrenos ubicados dentro de los centros de población donde se generan los residuos que procesan, sin riesgo de inundación, deslave o hundimiento y con vías de acceso en buen estado, cumpliendo con lo reportado como conveniente en la literatura [11]. 
Cuadro 1. Región socioeconómica y años de operación de los CRRV estudiados

\begin{tabular}{|c|c|c|}
\hline CRRV & Región socioeconómica & $\begin{array}{c}\text { Años en } \\
\text { operación }\end{array}$ \\
\hline 01 & Central (GAM) & 1 \\
\hline 02 & Central & 10 \\
\hline 03 & Central (GAM) & 3 \\
\hline 04 & Central (GAM) & 4 \\
\hline 05 & Chorotega & 9 \\
\hline 06 & Chorotega & 8 \\
\hline 07 & Central (GAM) & 6 \\
\hline 08 & Brunca & 2 \\
\hline 09 & Central (GAM) & 16 \\
\hline 10 & Central (GAM) & 10 \\
\hline 11 & Central (GAM) & 5 \\
\hline 12 & Central (GAM) & 13 \\
\hline 13 & Central (GAM) & 8 \\
\hline 14 & Central (GAM) & 10 \\
\hline 15 & Central & 12 \\
\hline
\end{tabular}

Por otro lado, un 53\% de las instalaciones estudiadas se encuentran en terrenos colindantes a casas de habitación y no poseen área de amortiguamiento entre las zonas de trabajo y las propiedades vecinas. Esto resulta inconveniente pues la acumulación de residuos cerca de las propiedades puede generar preocupaciones de índole sanitaria en los propietarios y provocar su oposición al proyecto [14]. En uno de los casos visitados se han recibido quejas de los vecinos por temor a la existencia de criaderos de dengue en los residuos acumulados. Adicionalmente, en un $60 \%$ de los terrenos no existe posibilidad de expandir el tamaño actual de las instalaciones.

\section{Infraestructura}

Como para toda actividad industrial, la infraestructura de los CRRV debe ser planificada y diseñada teniendo en cuenta la naturaleza de las actividades que se llevarán a cabo en el edificio, lo que incluye el diseño estructural, eléctrico y de fontanería [7]. En un 53\% de los casos estudiados, la infraestructura en que funcionan actualmente los CRRV no fue específicamente construida con ese fin, sino que corresponden a instalaciones en que anteriormente se desempeñaba otra clase de actividad, por lo cual sus características no están diseñadas para proveer una máxima eficiencia en la operación.

Las edificaciones en que operan los CRRV deben maximizar el espacio libre con el fin de proveer flexibilidad para el emplazamiento de los equipos y el tránsito interno. Sin embargo, en un 53\% de las edificaciones existen paredes internas que limitan el libre movimiento de residuos y operarios. Asimismo, la infraestructura debe permitir que el proceso fluya libremente sin retrocesos ni manejo repetido de los materiales, con una puerta para el acceso de residuos y otra para la salida del producto separado [11]. Contrario a esto, dos de las edificaciones visitadas poseen una única entrada, por lo que se produce interferencia entre la entrada y salida de residuos. Por otro lado, un $67 \%$ de las instalaciones no cuentan con un muelle para agilizar la operación de carga y descarga (figura 1). 
a)

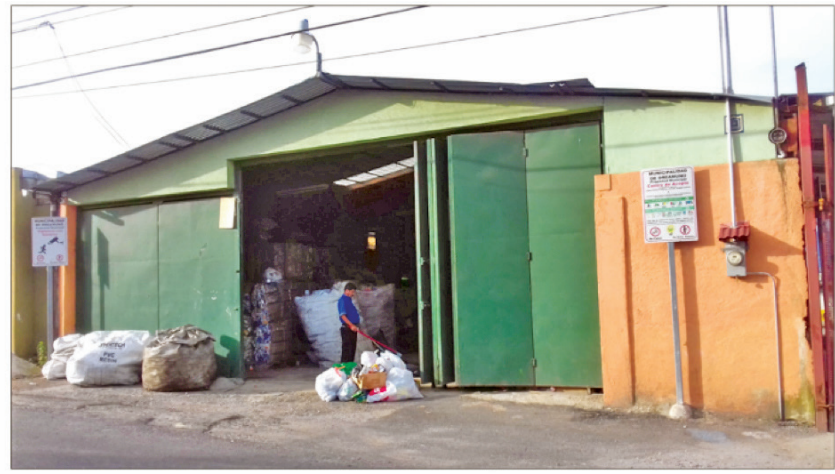

b)

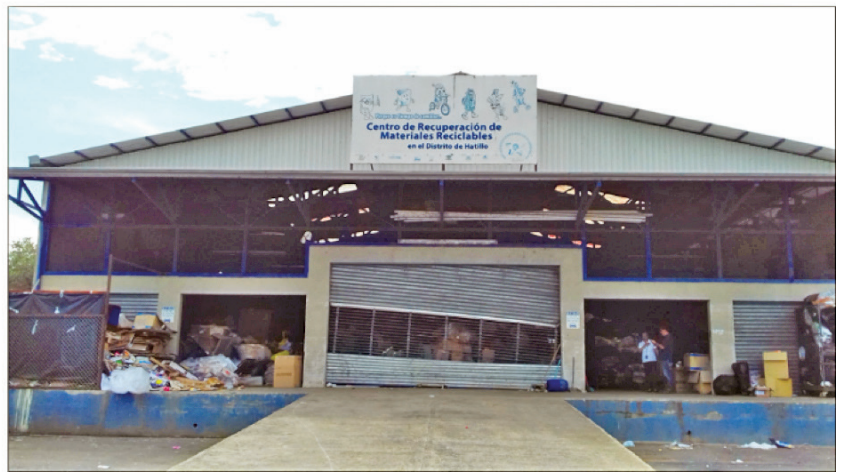

Figura 1. Instalación con un único acceso y sin muelle de carga (a) e instalación con entrada y salida separadas, así con como un muelle de carga (b).

\section{Gestión de materia prima}

\section{Cantidad de materiales vendidos}

Lobo et al. [15] utilizaron una clasificación de tamaño para los CRRV de Costa Rica basándose en la cantidad de toneladas de material vendido. Usando esta categorización los casos estudiados se agruparon según lo indicado en el cuadro 2. La única instalación que califica como Grande A es el CRRV 13, que de acuerdo con su jefe de planta es el CRRV de propiedad municipal de mayor tamaño del país [16].

Cuadro 2. Categorización de los CRRV estudiados con base en la cantidad de materiales vendidos.

\begin{tabular}{|c|c|c|}
\hline Categoría & Materiales vendidos (ton/mes) & Casos estudiados \\
\hline Pequeño & $<10$ & 5 \\
\hline Mediano & $10-30$ & 4 \\
\hline Grande B & $30-100$ & 1 \\
\hline Grande A & $>100$ & 4 \\
\hline
\end{tabular}




\section{Cantidad de residuos no recuperables}

No todos los residuos que ingresan a un centro de recuperación pueden ser efectivamente recuperados y comercializados, cierto porcentaje de la corriente de entrada debe desecharse y enviarse al relleno sanitario u otro sitio de disposición final, ya sea porque se trata de materiales para los cuales no se tiene comprador, materiales sucios o contaminados con materia orgánica, o porque son fracciones pequeñas de materiales valiosos que requerirían una inversión muy grande para su recuperación, como pueden ser trozos pequeños de vidrio.

La mayoría de los CRRV estudiados reportaron un porcentaje de residuos no recuperables de entre el $10 \%$ y el $25 \%$ del total que ingresa a las instalaciones (figura 2). El CRRV 06 y el 12 no realizaron una estimación numérica de la cantidad de residuos no recuperables, reportando el porcentaje como "muy pequeño".

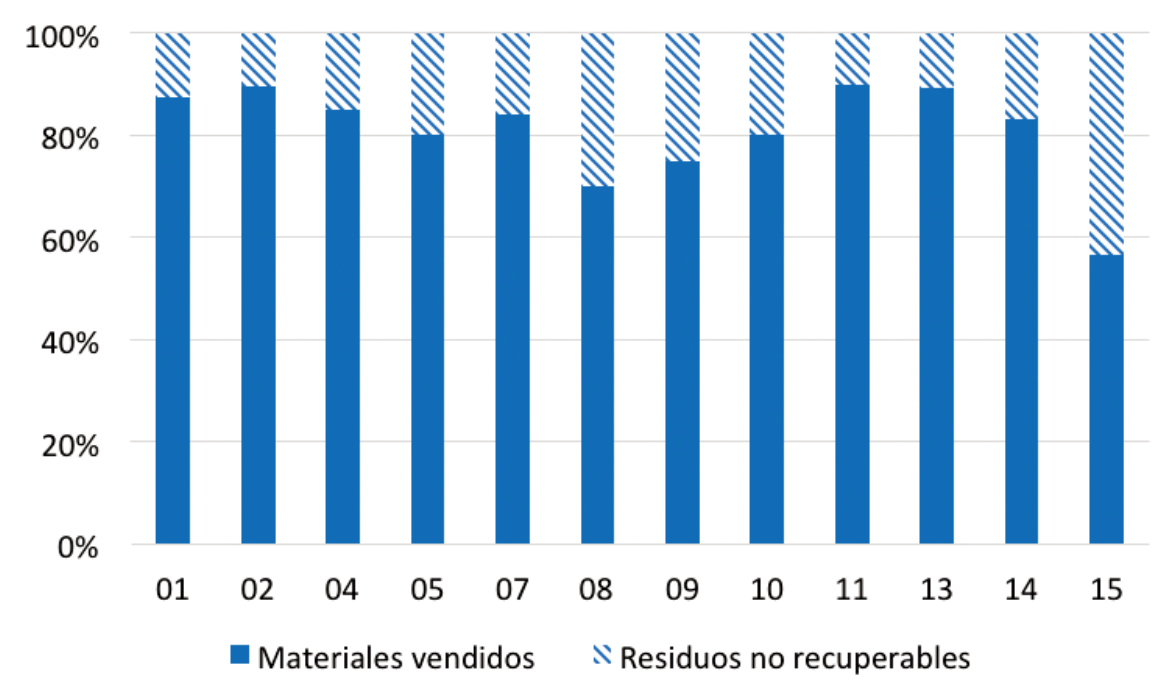

Figura 2. Porcentaje de residuos no recuperables en los CRRV estudiados

La tasa de valorización de residuos a nivel cantonal y nacional en Costa Rica es calculada con base en la cantidad de residuos recolectados por las municipalidades y entregados a los CRRV, sin tomar en consideración el porcentaje de residuos que no logra ser recuperado en estas instalaciones [17].

\section{Proveedores de residuos}

En todos los CRRV estudiados, los residuos que ingresan a las instalaciones provienen fundamentalmente de la recolección municipal de residuos valorizables, con excepción del CRRV 11 y el CRRV 14, que reciben únicamente residuos que ciudadanos particulares transportan a las instalaciones, modalidad conocida como Punto Limpio. En ninguno de los CRRV se paga por los materiales recibidos, sin embargo, cuatro de los casos estudiados participan del programa Ecolones, por lo que otorgan esta moneda virtual a cambio de los residuos que cumplan con los requisitos establecidos en el programa. 


\section{Descarga no autorizada de residuos}

La descarga no autorizada de residuos por parte de la población en la entrada de las instalaciones durante las noches o fines de semana es una situación común en los CRRV. La acumulación de estos residuos a la intemperie genera preocupaciones de índole ambiental como atracción de vectores y deterioro del paisaje [11]. Todos los encargados de los CRRV visitados manifestaron haberse encontrado en algún momento con esta situación.

Tipo de corriente de entrada

En la ENSRVR [5] se pretende armonizar la forma en que las fuentes clasifican los residuos que generan previo a que sean recolectados, proponiendo la separación en bolsas con distintivos de color según material. Sin embargo, en un 93\% de los CRRV estudiados los residuos nunca o casi nunca ingresan separados según lo propuesto en la ENSRVR, siendo lo normal que vengan en una sola bolsa los materiales mezclados. En los cuatro CRRV afiliados al programa Ecolones, se reciben residuos con mayor grado de separación cuando ciudadanos particulares los llevan con el fin de canjearlos por la moneda virtual. De acuerdo con los requisitos de Ecolones deben ser los usuarios quienes clasifiquen sus residuos.

\section{Tecnología para clasificación}

En lo referente a la operación de separación, ninguno de los CRRV estudiados utiliza equipos tecnológicos que aprovechen la diferencia en las propiedades físicas de los distintos materiales para su extracción de los residuos de entrada, la separación en todos los casos se realiza de manera completamente manual. Respecto a la superficie sobre la cual se realiza la clasificación de los residuos se encontró que en la mayoría de instalaciones esta operación se hace sobre mesas fijas (figura 3), pero también se encontraron casos en que se realiza la separación sobre el suelo y un CRRV, el número 10, en que se realiza sobre una banda transportadora (cuadro 3).

Cuadro 3. Forma de clasificar los materiales en los CRRV estudiados

\begin{tabular}{|c|c|}
\hline Forma de clasificar los materiales & Casos estudiados \\
\hline Manual sobre el suelo & 3 \\
\hline Manual sobre mesa & 11 \\
\hline Manual sobre banda transportadora & 1 \\
\hline
\end{tabular}

La clasificación manual sobre una superficie estática, suelo o mesa, es adecuada para procesar cantidades de residuos inferiores a las 2 toneladas por día, mientras que una banda transportadora de una sola línea permite aumentar el procesamiento al orden de 10 toneladas diarias [18]. Por lo tanto, la tecnología para clasificación con que cuentan los CRRV es adecuada para la cantidad de materiales procesados en un $87 \%$ de los casos.

Tecnología para procesamiento adicional de los materiales

En relación con el procesamiento adicional de los materiales, diez de los CRRV estudiados poseen equipos para compactar los residuos y nueve de ellos poseen máquinas para quebrar el vidrio. Esto quiere decir que una parte importante de los casos estudiados no puede acceder a los beneficios de aumentar la densidad de sus materiales procesados, lo cual incluye un mejor precio en el mercado y un menor requerimiento de espacio tanto para almacenamiento como transporte [11], [19]. 


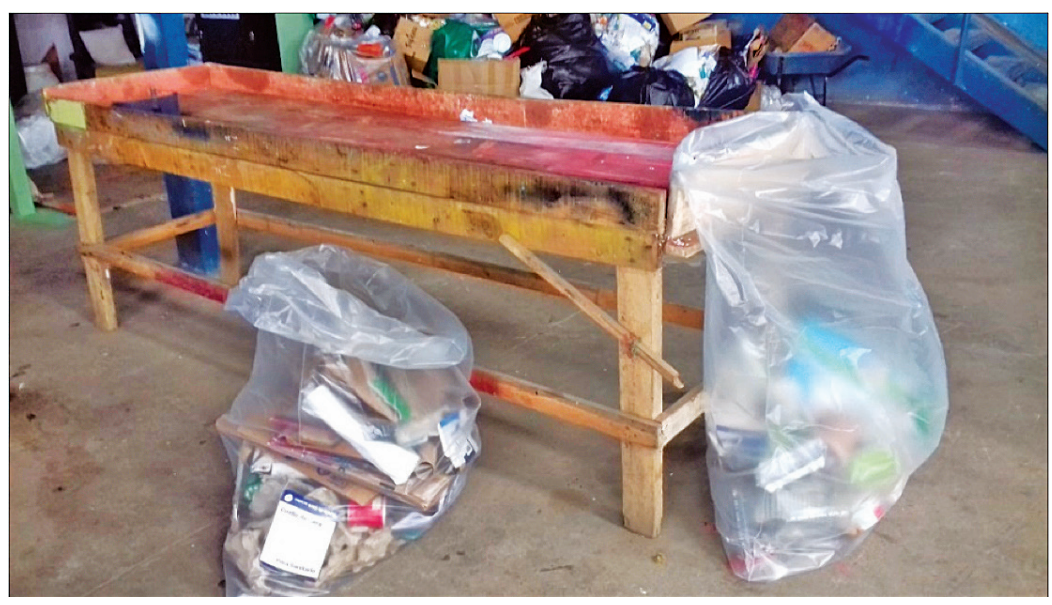

Figura 3. Mesa usada para la clasificación manual de residuos.

\section{Gestión del recurso humano}

\section{Cantidad y tipo de personal}

Los CRRV que dependen en gran medida de las actividades de clasificación manual son instalaciones intensivas en mano de obra [20]. Los CRRV municipales en Costa Rica son operados en algunos casos por personal contratado directamente por la municipalidad y en otras ocasiones por organizaciones comunales o empresas privadas con las que se establece un convenio. En el cuadro 4 se muestra la procedencia del personal operativo y la cantidad de trabajadores a tiempo completo con que cuentan las instalaciones. En algunas ocasiones los CRRV emplean personal temporal o voluntario, sin embargo, es difícil prever la disponibilidad de este personal [21], [22].

\section{Productividad del personal}

Un trabajador de una instalación en la que se emplea separación manual sobre una mesa alcanza una productividad promedio de $70 \mathrm{~kg}$ de material separado por día [7]. Durante la investigación se detectó la percepción generalizada, entre los administradores de los CRRV municipales, de que los empleados contratados por la municipalidad son menos productivos que los privados, pues su remuneración no depende de la cantidad de materiales procesados mientras que en los privados sí. La ausencia de registros de productividad y métodos cuantitativos para evaluar el desempeño de los operarios impidió corroborar la veracidad de esta afirmación. Sin embargo, fueron observadas durante las visitas de campo, una serie de errores en la gestión del personal, incluyendo falta de capacitación e inadecuadas condiciones de trabajo, que de acuerdo con la literatura repercuten en la productividad del personal y cuyo control es responsabilidad de la administración del CRRV [11], [23], [24],

Personal con limitaciones físicas y condiciones adversas de salud

Según Gómez [9] existe en algunas municipalidades la tendencia a utilizar el CRRV como forma de ocupar personal que, debido a limitaciones físicas o condiciones adversas de salud, se considera no puede desempeñarse en otras áreas como recolección de residuos o mantenimiento de vías. Esta tendencia fue confirmada en la investigación, donde en un 67\% de los CRRV estudiados se utilizan algunas veces este tipo de personal, incluyendo un $33 \%$ en que se usa casi siempre. 
Cuadro 4. Tipo y cantidad de personal permanente en los CRRV estudiados.

\begin{tabular}{|c|c|c|}
\hline CRRV & Procedencia del personal operativo & Operarios a tiempo completo \\
\hline 01 & Organización comunal & 2 \\
\hline 02 & Municipalidad & 5 \\
\hline 03 & Empresa privada & - \\
\hline 04 & Organización comunal & 4 \\
\hline 05 & Organización comunal & 14 \\
\hline 06 & Municipalidad & 1 \\
\hline 07 & Municipalidad & 2 \\
\hline 08 & Organización comunal & 18 \\
\hline 09 & Municipalidad & 35 \\
\hline 10 & Municipalidad & 4 \\
\hline 11 & Organización comunal & 2 \\
\hline 12 & Municipalidad & 21 \\
\hline 13 & Empresa privada & 6 \\
\hline 14 & Municipalidad & 6 \\
\hline 15 & Municipalidad & Empresa privada \\
\hline
\end{tabular}

De acuerdo con el jefe de planta de uno de los CRRV visitados, la asignación de este personal proviene de la creencia administrativa de que las operaciones en el CRRV no requieren de esfuerzo físico para desempeñarse eficientemente. Sin embargo, además de las labores de clasificación, que exigen permanecer en pie durante periodos prolongados de tiempo, se requiere de personal para labores de carga, descarga y estiba de pacas de residuos con un peso considerable [16]. El personal con limitaciones físicas puede ser asignado a labores de registro e inspección de calidad, requiriéndose contratar también personal en condiciones aptas para tareas de mayor exigencia física, esto con el fin de evitar situaciones de riesgo y contribuir a la ágil operación de las instalaciones.

\section{Capacitación}

La capacitación del personal constituye un factor clave en la eficiencia de la operación del CRRV [11]. Un ejemplo de esto ocurre cuando los operarios no están familiarizados con cierto tipo de material, pudiendo clasificarlo de manera incorrecta y contaminar toda una paca de producto [23]. En un 40\% de los CRRV estudiados no se da capacitación al personal que inicia labores por lo que este depende de observar a los trabajadores experimentados mientras trabajan, por lo cual su productividad es limitada durante los primeros días y son propensos a cometer errores.

En dos de los casos visitados la capacitación de los nuevos operarios es realizada por el Instituto Nacional de Aprendizaje (INA) mediante el curso Recuperador o Recuperadora de desechos sólidos. La duración del curso es de 98 horas, considerado muy extenso por parte de algunos administradores, lo que imposibilita la capacitación de sus operarios bajo esta modalidad. 


\section{Ergonomía de las estaciones de clasificación}

No se le puede exigir a un trabajador que rinda a su máximo potencial si las condiciones de trabajo no son las adecuadas. De acuerdo con lo recomendado en la literatura las estaciones de clasificación deben contar con suficiente ventilación e iluminación natural. Asimismo, las mesas de clasificación y los recipientes deben tener dimensiones y estar ubicados de tal manera que los trabajadores puedan manipular los residuos sin necesidad de estirarse o agacharse excesivamente, lo que redundaría en un mayor desgaste físico y una menor productividad [23], [24]. En un 60\% de los CRRV estudiados el diseño de las estaciones de clasificación resulta inadecuado en estos aspectos.

En varias de las instalaciones visitadas se observó como las bolsas utilizadas para clasificar los residuos tenían la apertura plegada (figura 4), además de estar a un nivel muy bajo, lo que provocaba que el operario debiera doblar la espalda y extender la apertura con sus manos cada vez que fuera a introducir un residuo.

a)



b)

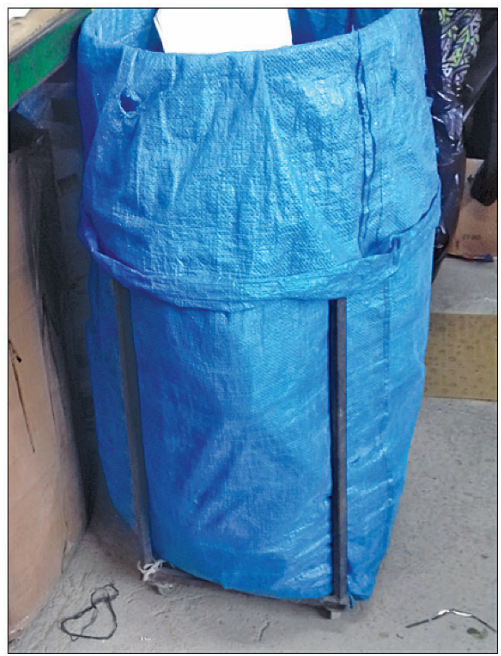

Figura 4. Recipientes con características inadecuadas (a) y adecuadas (b).

\section{Procedimientos de trabajo}

\section{Documentación}

Documentar un procedimiento consiste en llevar un registro escrito de los pasos necesarios para su correcta ejecución, estableciendo el personal responsable y los recursos que demanda. La documentación sirve como una guía de referencia para el personal y la administración. El proceso de documentación en sí contribuye a aumentar el conocimiento de la forma en que trabaja la organización, identificar las áreas de la operación susceptibles a mejora y enfocar las personas, recursos y tiempo al logro de objetivos y metas [25]. En un $47 \%$ de los CRRV analizados no se tienen procedimientos por escrito, mientras que en un $20 \%$ poseen pocos establecidos. En estos casos la forma de trabajo depende del personal disponible y su experiencia en la actividad a ejecutar. 
Almacenamiento de residuos al aire libre

Resulta siempre preferible almacenar el material procesado bajo techo, con el fin de evitar se dañe por efecto de la humedad, lo que reduciría su calidad, y que sea dispersado por el viento, causando contaminación [26]. Sin embargo, en ocasiones los CRRV pueden verse en la necesidad de almacenar material al aire libre debido a la limitada capacidad de almacenamiento de algunas instalaciones.

En caso de almacenar material al aire libre se deben tomar consideraciones especiales, como dar prioridad a los materiales con bajo tiempo de rotación y cubrir los residuos con lonas. En un CRRV visitado se observaron residuos almacenados al aire libre sin ninguna protección, afectando además la estética de las instalaciones (figura 5).

a)

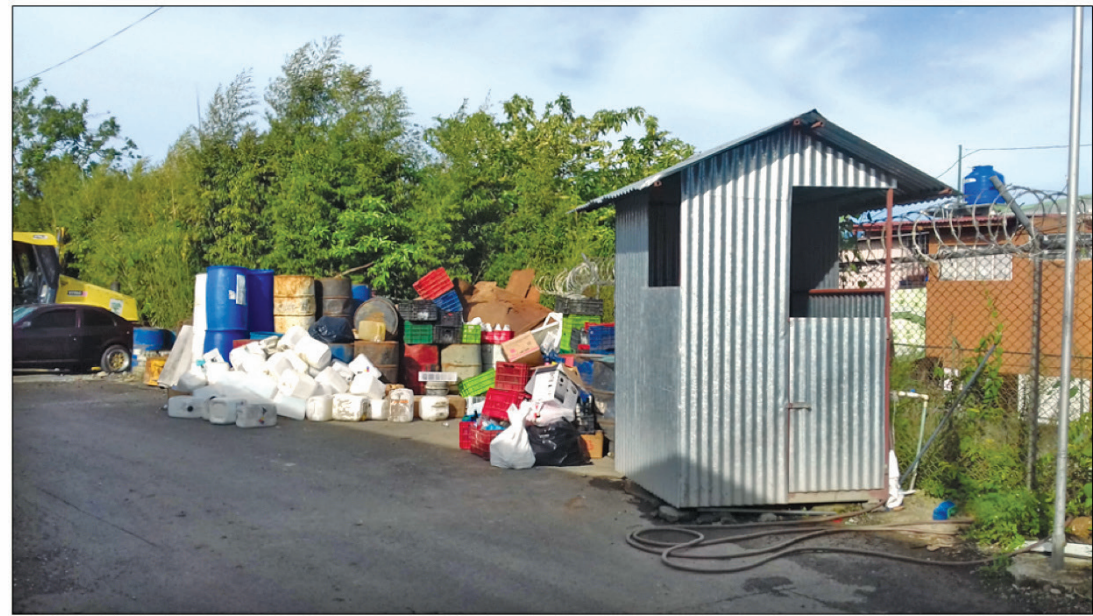

b)

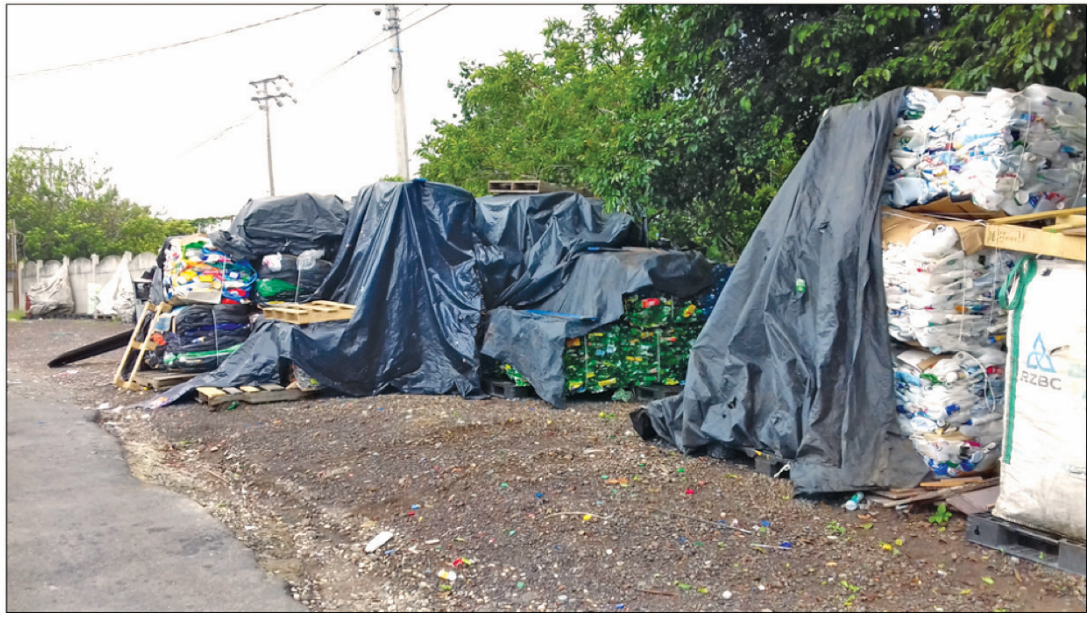

Figura 5. Inadecuado almacenamiento al aire libre (a) y adecuado (b).

\section{Formalidad de los contratos}

De acuerdo con Lobo et al., [15] las empresas recicladoras en el país no establecen contratos formales con sus proveedores, sino que basan la relación comercial en la confianza y la flexibilidad para negociar precios. Esto fue corroborado en el presente estudio ya que en ninguno de los CRRV analizados se establece un contrato formal con las empresas a las cuales se venden los materiales. 
Aunque la ausencia de un contrato formal con los compradores de material provee flexibilidad a los administradores de los centros de recuperación para adaptarse a cambios en las condiciones de mercado, también puede suceder que una empresa incumpla las condiciones del pacto verbal y comprometa la operación del CRRV. De acuerdo con la encargada del CRRV 11 [21] el material solía entregarse a un centro de recuperación privado, en una ocasión esa empresa dejó súbitamente de aceptar envíos debido a trabajos de reparación en el acceso a sus instalaciones, esto provocó problemas de acumulación de material en el CRRV municipal.

Control de calidad

Con el fin de generar confianza en el mercado los CRRV deben asegurar la calidad de los materiales que clasifican, para esto es necesario contar con algún mecanismo de control de calidad dentro de la organización, como puede ser la inspección visual al final de cada línea de clasificación o el muestreo al azar de los materiales empacados [27]. En un 60\% de los CRRV estudiados nunca o muy pocas veces se verifica la calidad de los materiales separados que se envían a las empresas recicladoras. La falta de verificación de la calidad de los envíos puede llegar a provocar un perjuicio importante para los CRRV. De acuerdo con el administrador de un CRRV entrevistado en una ocasión al llegar a la empresa recicladora se le ofreció pagar una paca de papel blanco con una tira de color como si toda la paca fuera de color, para evitar entregar la paca a un menor precio fue necesario llevársela y sacar la tira [19].

\section{Conclusiones}

La ubicación, topografía y accesibilidad de los terrenos en que se ubican los CRRV estudiados son en general adecuadas y no afectan negativamente el desempeño de las instalaciones.

En la mayoría de los casos la infraestructura de las edificaciones resulta inadecuada, pues no fue diseñada para funcionar como un CRRV, por lo que sus características no están orientadas a una máxima eficiencia en la operación.

La tecnología para clasificación con que cuentan los CRRV no representa una limitante considerable para el eficiente procesamiento de la cantidad de residuos que reciben.

Fueron observadas durante las visitas de campo, una serie de errores en la gestión del personal, incluyendo falta de capacitación e inadecuadas condiciones de trabajo, que repercuten negativamente en la productividad lo trabajadores y cuyo control es responsabilidad de la administración del CRRV.

No existe en general la práctica de documentar procesos en los CRRV analizados, la forma de trabajo depende del personal disponible y su experiencia en la actividad a ejecutar.

Los CRRV carecen de contratos formales con las empresas a las cuales venden los materiales lo que provoca inestabilidad en la planificación comercial.

No existe a nivel nacional una guía de buenas prácticas que aborde todas las etapas del diseño y la operación de los centros municipales de recuperación de residuos sólidos valorizables y sirva como referencia a los encargados para diseñar y operar instalaciones eficientemente.

\section{Recomendaciones}

Realizar investigación adicional enfocada en los CRRV municipales ubicados en otras regiones socioeconómicas del país más allá de la región central, con el fin de validar y complementar los resultados de la presente investigación. 
Contratar personal especializado para la gerencia y supervisión de los CRRV municipales.

En procura de mejorar el desempeño de la operación, se debe iniciar por entender y registrar la forma actual de trabajo, por lo que se recomienda documentar los procesos.

Establecer convenios entre las municipalidades, organizaciones gremiales y universidades públicas para compartir capacitación y asistencia técnica en el diseño y la operación de los CRRV.

Para el cálculo de la tasa de valorización a nivel cantonal y nacional se deben considerar como valorizados los residuos al momento de su envío a las empresas recicladoras y no al momento de su recepción en el CRRV, pues parte de los materiales que ingresan a un CRRV terminan de todas maneras en un sitio de disposición final.

Desarrollar una guía de buenas prácticas que incluya al menos recomendaciones concernientes a la escogencia del terreno donde ubicar los CRRV, características de la infraestructura, escogencia del equipo, gestión de materia prima, gestión de personal y procedimientos de trabajo.

\section{Referencias}

[1] A. Troschinetz.(2005). Twelvefactorsinfluencingsustainable recycling of municipal solid waste in developing countries [En línea]. Disponible: https://digitalcommons. mtu.edu/cgi/viewcontent.cgi?article=1276\&context=etds

[2] C. Cimpan, A. Maul, M. Jansen, T. Pretz and H. Wenzel. (2015, Abr.). "Central sorting and recovery of MSW recyclable materials: A review of technological state-of-the-art, cases, practice and implications for materials recycling. Journal of Environmental Management, 156, pp. 181-199. Doi:10.1016/j.jenvman.2015.03.025

[3] S. Zafar. (2017, Nov. 29). Introduction to MRF [En línea]. Disponible: https://www.ecomena.org/materialsrecovery-facility/

[4] "Ley N 8839. Ley para la Gestión Integral de Residuos Sólidos." Diario Oficial La Gaceta №135, 13 Julio 2010.

[5] Ministerio de Salud. (2016, Abr.). Estrategia Nacional de Separación, Recuperación y Valorización de Residuos (ENSRVR) [En línea]. Disponible: https://goo.gl/mB87FJ

[6] L.D. Jiménez, Comunicación personal, Fundación Aliarse, junio 2018.

[7] H. K. T. Campos. (2014). "Recycling in Brazil: Challenges and prospects". Resources, Conservation and Recycling, 85, pp. 130-138. Doi: 10.1016/j.resconrec.2013.10.017

[8] M. Torres, Comunicación personal, setiembre 2017.

[9] G. Gómez, Comunicación personal, setiembre 2017.

[10] M. López, Comunicación personal, octubre 2017.

[11] Illinois Recycling Association. (2010, Nov.). Best Operational Practices Manual for Materials Recovery Facilities and Recycling Drop-off Facilities [En línea]. Disponible: http://www.illinoisrecycles.org/wp-content/ uploads/2014/10/IRA_BOPM_2010.pdf

[12] M. Pérez. (2016, Mar. 25). Las áreas funcionales de la empresa [En línea]. Disponible: http://publicacionesdidacticas.com/hemeroteca/articulo/069027/articulo-pdf

[13] B. Richers, C. Harvey, F. Casanoves, F. DeClerck and T. Benjamin. "¿Cómo hacer talleres participativos con respuestas individuales?” Agroforestería en las Américas, 48, pp. 157 - 163, Ene. 2011

[14] P. Grandela, I. Honold and P. Mansilla. (2007, Nov.). Guía de Buenas Prácticas Ambientales para Instalaciones de Acopio de Chatarra [En línea]. Disponible: https://goo.gl/7HbLaE

[15] S. Lobo, M. Marín. V. Rudín and F. Salas. (2016). Análisis de los retos para el desarrollo de la Cadena de Valor del Reciclaje en Centroamérica [En línea]. Disponible: https://publications.iadb.org/bitstream/handle/11319/8102/Analisis-de-los-retos-para-el-desarrollo-de-la-cadena-de-valor-del-reciclaje-en-Centroamerica. pdf?sequence $=1$

[16] A. Valerín, Comunicación personal, junio 2018.

[17] E. Androvetto, Comunicación personal, mayo 2018. 
[18] Asian Development Bank. (2013, Abr.). Materials Recovery Facility Tool Kit [En línea]. Disponible: https://www. adb.org/publications/materials-recovery-facility-tool-kit.

[19] L. Meléndez, Comunicación personal, agosto 2017.

[20] United Nations Environment Programme (2017). Asia Waste Management Outlook [En línea]. Disponible: https://goo.gl/VH958N.

[21] G. Villalobos; Comunicación personal, mayo 2018.

[22] W. Brenes, Comunicación personal, junio 2018.

[23] M. Jones. (1992). Times and motion analyses of manual sorting procedures at materials recovery facilities [En línea]. Disponible: http://www.seas.columbia.edu/earth/wtert/sofos/nawtec/1992-National-Waste-ProcessingConference/1992-National-Waste-Processing-Conference-24.pdf

[24] United States Environmental Protection Agency. (1995, Ago). MITE Program Evaluation: Environmental, Economic and Energy Impacts of Material Recovery Facilities [En línea]. Disponible: https://goo.gl/4nuAfP

[25] F. Alzate. (2011, Feb. 23). Importancia de la documentación de un sistema de calidad [En línea]. Disponible: http://iso9001-calidad-total.com/importancia-de-la-documentacion-de-un-sistema-de-calidad/

[26] Agencia de Residuos de Cataluña. (2012, May.). Guía de Buenas Prácticas para el Reciclaje y la Recuperación de Papel y Cartón en Cataluña [En línea]. Disponible: http://residus.gencat.cat/web/.content/home/lagencia/ publicacions/prevencio/guiapapercartro_web_es.pdf

[27] Waste and Resources Action Programme. (2006, Sep.). MRFs Comparison of Efficiency and Quality [En línea]. Disponible: http://www.wrap.org.uk/sites/files/wrap/MRF_v6_19Dec06_LC.pdf 